\title{
The Primitive Derivation and Discrete Integrals
}

\author{
Daisuke SUYAMA a and Masahiko YOSHINAGA ${ }^{\mathrm{b}}$
}

a) Faculty of Integrated Media, Wakkanai Hokusei Gakuen University, 1-2290-28 Wakabadai, Wakkanai, Hokkaido 097-0013, Japan

E-mail: suyama@wakhok.ac.jp

b) Department of Mathematics, Faculty of Science, Hokkaido University, Kita 10, Nishi 8, Kita-Ku, Sapporo 060-0810, Japan

E-mail:yoshinaga@math.sci.hokudai.ac.jp

Received September 30, 2020, in final form April 09, 2021; Published online April 13, 2021 https://doi.org/10.3842/SIGMA.2021.038

\begin{abstract}
The modules of logarithmic derivations for the (extended) Catalan and Shi arrangements associated with root systems are known to be free. However, except for a few cases, explicit bases for such modules are not known. In this paper, we construct explicit bases for type $A$ root systems. Our construction is based on Bandlow-Musiker's integral formula for a basis of the space of quasiinvariants. The integral formula can be considered as an expression for the inverse of the primitive derivation introduced by K. Saito. We prove that the discrete analogues of the integral formulas provide bases for Catalan and Shi arrangements.
\end{abstract}

Key words: hyperplane arrangements; freeness; Catalan arrangements; Shi arrangements 2020 Mathematics Subject Classification: 52C35; 20F55

Dedicated to Professor Kyoji Saito on the occasion of his rrth birthday

\section{Introduction}

\section{$1.1 \quad$ Background}

Let $V$ be an $\ell$-dimensional linear space over $\mathbb{C}$. Let $S=S\left(V^{*}\right)$ be the set of polynomial functions on $V$. Choose $x_{1}, \ldots, x_{\ell}$ as a basis of $V^{*}$ and identify $S$ with the polynomial ring $\mathbb{C}\left[x_{1}, \ldots, x_{\ell}\right]$. Let $\operatorname{Der}_{S}=\bigoplus_{i=1}^{\ell} S \partial_{i}$ be the module of polynomial vector fields ( $\mathbb{C}$-linear derivations of $S$ ).

Let $\mathcal{A}=\left\{H_{1}, \ldots, H_{n}\right\}$ be a central arrangement of hyperplanes. For each $H \in \mathcal{A}$, choose a linear form $\alpha_{H} \in V^{*}$ such that $H=\operatorname{Ker}\left(\alpha_{H}\right)$. For each nonnegative integer $m$, let

$$
D(\mathcal{A}, m):=\left\{\delta \in \operatorname{Der}_{S} \mid \delta \alpha_{H} \in\left(\alpha_{H}^{m}\right) \text {, for any } H \in \mathcal{A}\right\} .
$$

If $m=1, D(\mathcal{A}, 1)$ is simply denoted by $D(\mathcal{A})$, whose elements are called logarithmic derivations. The module $D(\mathcal{A})$ was introduced in [13] for the purpose of computing Gauss-Manin connections, and $D(\mathcal{A}, m)$ was introduced by Ziegler [26] for studying restrictions of free arrangements. The algebraic structures of these modules are thought to reflect the combinatorial nature of $\mathcal{A}$ (see $[11,25])$.

Now we assume that $\mathcal{A}$ is a Coxeter arrangement, that is, the set of reflecting hyperplanes of a finite irreducible real reflection group $W \subset \mathrm{GL}(V)$. The definition of $D(\mathcal{A}, m)$ naturally gives rise to a filtration:

$$
\operatorname{Der}_{S}=D(\mathcal{A}, 0) \supset D(\mathcal{A}, 1) \supset D(\mathcal{A}, 2) \supset \cdots .
$$

This paper is a contribution to the Special Issue on Primitive Forms and Related Topics in honor of Kyoji Saito for his 77th birthday. The full collection is available at https://www.emis.de/journals/SIGMA/Saito.html 
The filtration (1.1) is closely related to several important structures. First, taking $W$-invariant parts, we have

$$
D(\mathcal{A}, 0)^{W}=D(\mathcal{A}, 1)^{W} \supset D(\mathcal{A}, 2)^{W}=D(\mathcal{A}, 3)^{W} \supset \cdots .
$$

This filtration is known to be equal to the semi-infinite Hodge filtration studied by K. Saito [15, $16,21,23]$, which is a crucial structure in his theory of primitive forms [14]. In particular, the inverse operator of the so-called primitive derivation $\nabla_{D}$ describes the filtration as

$$
D(\mathcal{A}, 2 m+1)^{W}=\left(\nabla_{D}^{-1}\right)^{m} D(\mathcal{A})^{W} .
$$

As indicated by Misha Feigin (see forthcoming paper [1] for details), these spaces are also isomorphic to the isotypic component of the spaces of $m$-quasiinvariants, which were introduced in the study of the Calogero-Moser system $[4,5,8]$.

Around 2000, Terao proved that the module $D(\mathcal{A}, m)$ is an $S$-free module using these structures [20]. Terao's results on the freeness of $D(\mathcal{A}, m)$ opened new perspectives between the primitive derivation and enumerative combinatorics of Catalan/Shi arrangements.

Catalan and Shi arrangements are classes of finite truncations of affine Weyl arrangements for root systems. The terminology "Catalan arrangement" is explained by the fact that the number of chambers in the fundamental region of a type $A$ root system is equal to the Catalan number [12]. The Shi arrangement was introduced by J.-Y. Shi in [17] in the study of affine Weyl groups. In 1996, Edelman-Reiner [6] posed a conjecture concerning the freeness of cones of Catalan and Shi arrangements for root systems. This conjecture was proved for type $A$ root system by Edelman-Reiner [6] and Athanasiadis [3], and later for all root systems by [24]. The freeness of $D(\mathcal{A}, m)$ played crucial role in the proof of [24] because $D(\mathcal{A}, m)$ can be regarded as the "leading terms" of the logarithmic vector fields for Catalan and Shi arrangements. In the present paper we focus on the construction of explicit bases for these modules.

\subsection{Constructions of explicit bases}

In this section, we introduce Catalan and Shi arrangements (of type $A_{\ell-1}$ ). We define $m$-Catalan arrangement $\mathrm{Cat}_{\ell}(m)$ as

$$
\prod_{\substack{1 \leq i<j \leq \ell \\-m \leq k \leq m}}\left(x_{i}-x_{j}-k\right)=0,
$$

and $m$-Shi arrangement $\operatorname{Shi}_{\ell}(m)$ by

$$
\prod_{1-m \leq k \leq m} \prod_{1 \leq i<j \leq \ell}\left(x_{i}-x_{j}-k\right)=0
$$

We also denote $\operatorname{Cat}_{\ell}(0)$ by $\mathcal{B}_{\ell}$. The arrangement $\mathcal{B}_{\ell}$ is defined as the polynomial $\prod_{1 \leq i<j \leq \ell}\left(x_{i}-x_{j}\right)$ and called the braid arrangement.

The cones $c \operatorname{Cat}_{\ell}(m)$ and $c \operatorname{Shi}_{\ell}(m)$ are defined by the homogeneous polynomials

$$
z \prod_{\substack{1 \leq i<j \leq \ell \\-m \leq k \leq m}}\left(x_{i}-x_{j}-k z\right)=0,
$$

and

$$
z \prod_{1-m \leq k \leq m} \prod_{1 \leq i<j \leq \ell}\left(x_{i}-x_{j}-k z\right)=0
$$



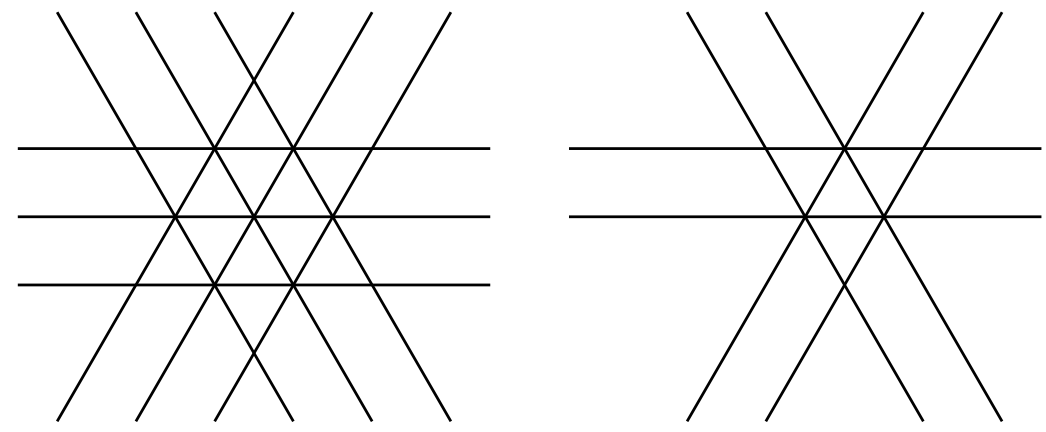

Figure 1. $\operatorname{Cat}_{3}(1)$ and $\operatorname{Shi}_{3}(1)$ (the intersections with the plane $x_{1}+x_{2}+x_{3}=0$ are drawn).

respectively. As we have already noted, the modules $D\left(c \mathrm{Cat}_{\ell}(m)\right)$ and $D\left(c \operatorname{Shi}_{\ell}(m)\right)$ are free. The exponents, that is, the degrees of the homogeneous bases of the modules, are as follows

$$
\begin{aligned}
& \exp \left(c \operatorname{Cat}_{\ell}(m)\right)=\{0,1, m \ell+1, m \ell+2, \ldots, m \ell+\ell-1\}, \\
& \exp \left(c \operatorname{Shi}_{\ell}(m)\right)=\{0,1, \underbrace{m \ell, m \ell, \ldots, m \ell}_{\ell-1}\} .
\end{aligned}
$$

We note that explicit bases were not constructed in the known proofs. Indeed, the proofs by Edelman-Reiner [6] and Athanasiadis [3] used Terao's addition-deletion theorem of freeness [11, Theorem 4.51], and that in [24] used cohomological arguments to guarantee the existence of global sections of certain coherent sheaves associated with the graded module $D(\mathcal{A})$. Since then, a number of efforts have been made to construct explicit bases for $D\left(c \mathrm{Cat}_{\ell}(m)\right)$ and $D\left(c \operatorname{Shi}_{\ell}(m)\right)$. First, in [19], a basis for $D\left(c \mathrm{Shi}_{\ell}(1)\right)$ was constructed using the Bernoulli polynomial. Subsequently, in [10] and [18], similar bases were constructed for root systems of type $B, C$, and $D$. Note that these works are for Shi arrangements with $m=1$. Catalan arrangements and Shi arrangements with $m>1$ have not been covered. For larger $m$, the type $A_{2}$ was the only known case. Namely, explicit bases were constructed for $c \operatorname{Cat}_{3}(m)$ and $c \mathrm{Shi}_{3}(m), m \geq 1$, in [2].

Our purpose in the present paper is to construct an explicit basis for $D\left(c \operatorname{Cat}_{\ell}(m)\right)$ and $D\left(c \operatorname{Shi}_{\ell}(m)\right)$, for all $\ell \geq 2$ and $m \geq 1$. The paper is organized as follows. The starting point of our study is Bandlow-Musiker's integral expression [4] (which goes back to FelderVeselov's integral expression [9]) for a basis of the space of quasiinvariants introduced in [5, 8]. Misha Feigin [7] communicated to us that Bandlow-Musiker's formula provides a basis for the multiarrangement $D\left(\mathcal{B}_{\ell}, 2 m+1\right)$. More precisely, for appropriate choices of $k$, the integral expressions

$$
\sum_{i, j=1}^{\ell}\left(\int_{x_{i}}^{x_{j}} t^{k}\left(\prod_{p=1}^{\ell}\left(t-x_{p}\right)\right)^{m} \mathrm{~d} t\right) \partial_{i}
$$

provide a basis for $D\left(\mathcal{B}_{\ell}, 2 m+1\right)$. We discuss these facts in addition to a basis for $D\left(\mathcal{B}_{\ell}, 2 m\right)$ in Section 2.

After introducing the notion of "discrete integrals" in Section 3, we present the main results in Section 4, that is, we prove the following: a basis for $D\left(c\right.$ Cat $\left._{\ell}(m)\right)$ is obtained from (1.2) by simply replacing the integration " $\int_{a}^{b} \mathrm{~d} t$ " with the discrete integration " $\sum_{a}^{b} \Delta t$ " as follows

$$
\sum_{i, j=1}^{\ell}\left(\sum_{x_{i}}^{x_{j}} t^{k}\left(\prod_{p=1}^{\ell}\left(t-x_{p}\right)\right)^{\underline{m}} \Delta t\right) \partial_{i} .
$$

(To be precise, we need to homogenize the above polynomial vector field, see Sections 3 and 4 for notations and details.) We also provide a basis for $D\left(c \mathrm{Shi}_{\ell}(m)\right)$. 


\section{Bandlow-Musiker's expression}

Recall that $\mathcal{B}_{\ell}$ denotes the braid arrangement defined by $\prod_{1 \leq i<j \leq \ell}\left(x_{i}-x_{j}\right)$. The symmetric group $W=\mathfrak{S}_{\ell}$ naturally acts on $\mathcal{B}_{\ell}$ by the permutation of coordinates. In this section, we construct a basis for $D\left(\mathcal{B}_{\ell}, m\right), m \geq 1$. Note that because the vector field

$$
\theta_{0}:=\partial_{1}+\partial_{2}+\cdots+\partial_{\ell}
$$

annihilates the linear form $x_{i}-x_{j}$, we have $\theta_{0} \in D\left(\mathcal{B}_{\ell}, m\right)$ for any $m$.

We also set $g(t):=\left(t-x_{1}\right)\left(t-x_{2}\right) \cdots\left(t-x_{\ell}\right) \in \mathbb{C}\left[t, x_{1}, \ldots, x_{\ell}\right]$. Following Bandlow-Musiker [4] and Feigin [7], for $m, k \geq 0$, we introduce the following vector field

$$
\eta_{k}^{m}=\sum_{i=1}^{\ell}\left(\sum_{j=1}^{\ell} \int_{x_{i}}^{x_{j}} t^{k} g(t)^{m} \mathrm{~d} t\right) \partial_{i} .
$$

Proposition $2.1([4,7])$. The vector fields $\eta_{0}^{m}, \eta_{1}^{m}, \ldots, \eta_{\ell-2}^{m}, \theta_{0}$ form a basis for $D\left(\mathcal{B}_{\ell}, 2 m+1\right)^{W}$ as an $S^{W}$-module.

Recall that Terao [20] proved that there exists a $W$-invariant basis for $D\left(\mathcal{B}_{\ell}, 2 m+1\right)$. Terao's invariant basis generates a submodule of $D\left(\mathcal{B}_{\ell}, 2 m+1\right)^{W}$ over $S^{W}$. However, these must coincide since the degrees of Terao's basis are equal to those of the above basis. Thus we have the following.

Corollary 2.2. The vector fields $\eta_{0}^{m}, \eta_{1}^{m}, \ldots, \eta_{\ell-2}^{m}, \theta_{0}$ form a basis of $D\left(\mathcal{B}_{\ell}, 2 m+1\right)$ as an $S$ module.

Here we give a direct proof of Corollary 2.2 in order to see the relationship between integral expression (2.1) and the primitive derivation. First, we prove $\eta_{k}^{m} \in D\left(\mathcal{B}_{\ell}, 2 m+1\right)$. Since $\eta_{k}^{m}$ is $W$-symmetric, it is sufficient to show that

$$
\eta_{k}^{m}\left(x_{1}-x_{2}\right)=\ell \int_{x_{1}}^{x_{2}} t^{k} g(t)^{m} \mathrm{~d} t
$$

is divisible by $\left(x_{1}-x_{2}\right)^{2 m+1}$. This can be checked by the change of variables $t^{\prime}=t-x_{1}$. Indeed, since the integrand is divisible by $\left(t-x_{1}\right)^{m}\left(t-x_{2}\right)^{m}$, after the change of variable, it is divisible by $\left(t^{\prime}\right)^{m}\left(t^{\prime}-\left(x_{2}-x_{1}\right)\right)^{m}$. Therefore, the definite integral $\int_{0}^{x_{2}-x_{1}} \mathrm{~d} t^{\prime}$ is divisible by $\left(x_{2}-x_{1}\right)^{2 m+1}$.

Example 2.3. When $\ell=2, m=1$, we obtain the following famous formula

$$
\int_{x_{1}}^{x_{2}}\left(t-x_{1}\right)\left(t-x_{2}\right) \mathrm{d} t=\frac{\left(x_{1}-x_{2}\right)^{3}}{6} .
$$

Next we describe the action of the primitive derivation on the vector field $\eta_{k}^{m}$. Let $\nabla$ denote the integrable connection with flat sections $\partial_{1}, \ldots, \partial_{\ell}$. More explicitly, for polynomial vector fields $\delta$ and $\eta=\sum_{i=1}^{\ell} f_{i} \partial_{i}$, we define

$$
\nabla_{\delta} \eta=\sum_{i=1}^{\ell}\left(\delta f_{i}\right) \partial_{i}
$$

Let $P_{i}$ denote the coefficient of $t^{\ell-i}$ in $g(t)$, that is, $g(t)=t^{\ell}+P_{1} t^{\ell-1}+\cdots+P_{\ell}$. Then $P_{1}, \ldots, P_{\ell} \in \mathbb{Z}\left[x_{1}, \ldots, x_{\ell}\right]$ are elementary symmetric functions (up to the sign), and satisfy $\mathbb{C}\left[x_{1}, \ldots, x_{\ell}\right]^{W}=\mathbb{C}\left[P_{1}, \ldots, P_{\ell}\right]$. Therefore, we can regard $P_{1}, \ldots, P_{\ell}$ as a system of coordinates 
on the quotient space $\mathbb{C}^{\ell} / W$. The vector field $D=\frac{\partial}{\partial P_{\ell}}$ is called the primitive derivation $[15,16]$. The lift of $D$ by the natural projection $\pi: \mathbb{C}^{\ell} \longrightarrow \mathbb{C}^{\ell} / W$ is expressed as

$$
D=\frac{1}{Q} \operatorname{det}\left(\begin{array}{ccccc}
\frac{\partial P_{1}}{\partial x_{1}} & \frac{\partial P_{2}}{\partial x_{1}} & \ldots & \frac{\partial P_{\ell-1}}{\partial x_{1}} & \frac{\partial}{\partial x_{1}} \\
\frac{\partial P_{1}}{\partial x_{2}} & \frac{\partial P_{2}}{\partial x_{2}} & \ldots & \frac{\partial P_{\ell-1}}{\partial x_{2}} & \frac{\partial}{\partial x_{2}} \\
\vdots & \vdots & \ddots & \vdots & \vdots \\
\frac{\partial P_{1}}{\partial x_{\ell}} & \frac{\partial P_{2}}{\partial x_{\ell}} & \ldots & \frac{\partial P_{\ell-1}}{\partial x_{\ell}} & \frac{\partial}{\partial x_{\ell}}
\end{array}\right),
$$

where $Q=\operatorname{det}\left(\frac{\partial P_{i}}{\partial x_{j}}\right)$ is the Jacobian of the projection $\pi$. For simplicity, we also denote by $D$ the lift of the primitive derivation $\frac{\partial}{\partial P_{\ell}}$. Note that other vector fields $\frac{\partial}{\partial P_{1}}, \frac{\partial}{\partial P_{2}}, \ldots, \frac{\partial}{\partial P_{\ell-1}}$ also have similar expressions. The definition (2.1) can be written as

$$
\eta_{k}^{m}=\sum_{i=1}^{\ell}\left(\sum_{j=1}^{\ell} \int_{x_{i}}^{x_{j}} t^{k}\left(t^{\ell}+P_{1} t^{\ell-1}+\cdots+P_{\ell}\right)^{m} \mathrm{~d} t\right) \partial_{i} .
$$

Then, if $m>0$, differentiation by $\frac{\partial}{\partial P_{j}}$ yields (see Remark 2.5 for details)

$$
\nabla_{\frac{\partial}{\partial P_{j}}} \eta_{k}^{m}=m \eta_{k+\ell-j}^{m-1}
$$

When $m=0$, it is checked by Saito's criterion $[11,13]$ that the vector fields $\eta_{0}^{0}, \eta_{1}^{0}, \ldots, \eta_{\ell-2}^{0}, \theta_{0}$ form a basis of $D\left(\mathcal{B}_{\ell}, 1\right)$. For $m>0$, we have $\nabla_{D}^{m} \eta_{k}^{m}=m ! \cdot \eta_{k}^{0}$. Recall that $D\left(\mathcal{B}_{\ell} \cap H_{0}\right.$, $2 m+1)^{W}=\nabla_{D}^{-m} D\left(\mathcal{B}_{\ell} \cap H_{0}, 1\right)^{W}\left[23\right.$, Corollary 10]. Therefore, $\nabla_{D}^{-m} \eta_{0}^{0}, \ldots, \nabla_{D}^{-m} \eta_{\ell-1}^{0}$ form a basis of $D\left(\mathcal{B}_{\ell} \cap H_{0}, 2 m+1\right)$, where $H_{0}$ is the hyperplane $x_{1}+\cdots+x_{\ell}=0$. Adding $\theta_{0}$, we obtain a basis for $D\left(\mathcal{B}_{\ell}, 2 m+1\right)$. This completes the proof of Corollary 2.2.

Now we consider $D\left(\mathcal{B}_{\ell}, 2 m\right)$. Let $g_{k}(t):=\frac{g(t)}{\left(t-x_{k}\right)}=\left(t-x_{1}\right) \cdots\left(\widehat{t-x_{k}}\right) \cdots\left(t-x_{\ell}\right)$ for $1 \leq k \leq \ell$. For $m>0$ and $k=1, \ldots, \ell$, we define the vector field $\sigma_{k}^{m}$ as

$$
\sigma_{k}^{m}:=\sum_{i=1}^{\ell}\left(\sum_{j=1}^{\ell} \int_{x_{i}}^{x_{j}} g(t)^{m-1} g_{k}(t) \mathrm{d} t\right) \partial_{i} .
$$

Proposition 2.4. Vector fields $\theta_{0}, \sigma_{1}^{m}-\sigma_{2}^{m}, \sigma_{2}^{m}-\sigma_{3}^{m}, \ldots, \sigma_{\ell-1}^{m}-\sigma_{\ell}^{m}$ form a basis of $D\left(\mathcal{B}_{\ell}, 2 m\right)$.

Proof. We first note that $\frac{\partial g(t)}{\partial x_{k}}=-g_{k}(t)$. Hence we have

$$
\nabla_{\partial_{k}} \eta_{0}^{m}=-m \sigma_{k}^{m}
$$

and

$$
\nabla_{\left(\partial_{i}-\partial_{i+1}\right)} \eta_{0}^{m}=-m\left(\sigma_{i}^{m}-\sigma_{i+1}^{m}\right) .
$$

Since $\partial_{1}-\partial_{2}, \ldots, \partial_{\ell-1}-\partial_{\ell}$ form a basis of $H_{0}$, by [23, Theorem 7$], \theta_{0}, \nabla_{\left(\partial_{1}-\partial_{2}\right)} \eta_{0}^{m}, \ldots, \nabla_{\left(\partial_{\ell-1}-\partial_{\ell}\right)} \eta_{0}^{m}$ form a basis of $D\left(\mathcal{B}_{\ell}, 2 m\right)$.

Remark 2.5. Recall that for certain functions $a(x), b(x), f(x, t)$, we have

$$
\frac{\mathrm{d}}{\mathrm{d} x} \int_{a(x)}^{b(x)} f(x, t) \mathrm{d} t=\frac{\mathrm{d} b(x)}{\mathrm{d} x} f(x, b(x))-\frac{\mathrm{d} a(x)}{\mathrm{d} x} f(x, a(x))+\int_{a(x)}^{b(x)} \frac{\partial f(x, t)}{\partial x} \mathrm{~d} t .
$$

In our setting, since the integrand $t^{k} g(t)^{m}$ vanishes at $x_{i}$, the first two terms do not contribute. Thus we have the equations (2.2) and (2.4). 


\section{Discrete integrals}

In this section we only consider polynomial functions. For a function $f(t)$, we define the difference operator $\Delta$ as $\Delta f(t)=f(t+1)-f(t)$. When $\Delta F(t)=f(t), F(t)$ is called an indefinite summation (or antidifference) of $f(t)$, and denoted by

$$
F(t)=\sum f(t) \Delta t
$$

Let $F(t)$ be an indefinite summation of $f(t)$. Then we define the definite summation as

$$
\sum_{a}^{b} f(t) \Delta t=F(b)-F(a) .
$$

Obviously we have the following.

$$
\begin{aligned}
& \sum_{b}^{a} f(t) \Delta t=-\sum_{a}^{b} f(t) \Delta t, \\
& \sum_{a}^{c} f(t) \Delta t=\sum_{a}^{b} f(t) \Delta t+\sum_{b}^{c} f(t) \Delta t .
\end{aligned}
$$

Note that if $b-a=n$ is a positive integer, the definite summation is nothing but the finite sum

$$
\sum_{a}^{b} f(t) \Delta t=f(a)+f(a+1)+\cdots+f(b-1) .
$$

Example 3.1. Recall that the Bernoulli polynomial $B_{n}(t)$ is a monic polynomial with rational coefficients defined by

$$
\sum_{n=0}^{\infty} \frac{B_{n}(x)}{n !} t^{n}=\frac{t \mathrm{e}^{x t}}{\mathrm{e}^{t}-1},
$$

(e.g., $B_{0}(x)=1, B_{1}(x)=x-\frac{1}{2}, B_{2}(x)=x^{2}-x+\frac{1}{6}, B_{3}(x)=x^{3}-\frac{3}{2} x^{2}+\frac{1}{2} x, B_{4}(x)=$ $\left.x^{4}-2 x^{3}+x^{2}-\frac{1}{30}, \ldots\right)$. By applying the difference operator with respect to the variable $x$ to the equation (3.2), we have

$$
\sum_{n=0}^{\infty} \frac{\Delta B_{n}(x)}{n !} t^{n}=t \mathrm{e}^{x t}=\sum_{n=1}^{\infty} \frac{x^{n-1}}{(n-1) !} t^{n} .
$$

Thus we have $\Delta B_{n}(t)=n t^{n-1}$. Therefore, the monomial $x^{n}$ has an indefinite summation $\frac{B_{n+1}(x)}{n+1}$. Furthermore, an arbitrary polynomial $f(x)=\sum_{i} a_{i} x^{i}$ has an indefinite summation $\sum_{i} a_{i} \frac{B_{i+1}(x)}{i+1}$.

The leading part of a definite summation is equal to a definite integral. More precisely, we have the following.

Proposition 3.2. Let $f\left(x_{0}, x_{1}, \ldots, x_{n}\right) \in \mathbb{C}\left[x_{0}, \ldots, x_{n}\right]$ be a homogeneous polynomial of degree d. Let

$$
F\left(y_{1}, y_{2}, x_{1}, \ldots, x_{n}\right):=\sum_{y_{1}}^{y_{2}} f\left(x_{0}, x_{1}, \ldots, x_{n}\right) \Delta x_{0}
$$

Then $F\left(y_{1}, y_{2}, x_{1}, \ldots, x_{n}\right)$ is a (not necessarily homogeneous) polynomial of degree $d+1$ in $y_{1}, y_{2}, x_{1}, \ldots, x_{n}$ whose highest degree part is

$$
\lim _{z \rightarrow 0} z^{d+1} F\left(\frac{y_{1}}{z}, \frac{y_{2}}{z}, \frac{x_{1}}{z}, \ldots, \frac{x_{n}}{z}\right)=\int_{y_{1}}^{y_{2}} f\left(x_{0}, x_{1}, \ldots, x_{n}\right) \mathrm{d} x_{0} .
$$

Proof. This is straightforward from the fact that the leading term of $\frac{B_{n+1}(t)}{n+1}$ is $\frac{t^{n+1}}{n+1}$, which is an indefinite integral of $t^{n}$.

The following is a discrete analogue of the power. Let $n>0$ be a positive integer. We define the falling power $f(t)^{\underline{n}}$ as

$$
f(t)^{\underline{n}}=f(t) f(t-1) \cdots f(t-n+1) .
$$




\section{Main results}

\subsection{A basis for the Catalan arrangement}

Let $\delta=\sum_{i=1}^{\ell} f_{i}\left(x_{1}, \ldots, x_{\ell}\right) \partial_{i}$ be a polynomial vector field $\left(f_{1}, \ldots, f_{\ell}\right.$ are not necessarily homogeneous). Let $d:=\max \left\{\operatorname{deg} f_{1}, \ldots, \operatorname{deg} f_{\ell}\right\}$. We define the homogenization of $\delta$ by

$$
\widetilde{\delta}=\sum_{i=1}^{\ell} z^{d} f_{i}\left(\frac{x_{1}}{z}, \ldots, \frac{x_{\ell}}{z}\right) \partial_{i} .
$$

Using the notion of discrete integrals, we define $\zeta_{k}^{m}$ as follows.

$$
\zeta_{k}^{m}=\sum_{i, j=1}^{\ell}\left(\sum_{x_{i}}^{x_{j}} t^{k} g(t)^{\underline{m}} \Delta t\right) \partial_{i} .
$$

Note that the definition of $\zeta_{k}^{m}$ is a discrete analogue of (2.1). The next result shows that the homogenizations of these vector fields form a basis for the Catalan arrangement.

Theorem 4.1. $\widetilde{\zeta}_{0}^{m}, \widetilde{\zeta}_{1}^{m}, \ldots, \widetilde{\zeta}_{\ell-2}^{m}, \theta_{0}$ and the Euler vector field $\theta_{E}:=z \partial_{z}+x_{1} \partial_{1}+\cdots+x_{\ell} \partial_{\ell}$ form a basis of $D\left(c \mathrm{Cat}_{\ell}(m)\right)$.

Proof. Note that the restriction of $c \mathrm{Cat}_{\ell}(m)$ to the hyperplane $z=0$ is equal to the braid arrangement $\mathcal{B}_{\ell}$ (with multiplicity $2 m+1$ ). In view of Ziegler's characterization of freeness [26] (see also [25, Corollary 1.35]), it is sufficient to prove the following:

(a) $\widetilde{\zeta}_{0}^{m}, \widetilde{\zeta}_{1}^{m}, \ldots, \widetilde{\zeta}_{\ell-2}^{m} \in D\left(c \operatorname{Cat}_{\ell}(m)\right)$.

(b) The restrictions $\left.\widetilde{\zeta}_{0}^{m}\right|_{z=0},\left.\widetilde{\zeta}_{1}^{m}\right|_{z=0}, \ldots,\left.\widetilde{\zeta}_{\ell-2}^{m}\right|_{z=0},\left.\theta_{0}\right|_{z=0}$ form a basis of $D\left(\mathcal{B}_{\ell}, 2 m+1\right)$.

First we prove $(b)$. By Proposition 3.2, we have

$$
\left.\widetilde{\zeta}_{0}^{m}\right|_{z=0}=\eta_{k}^{m}
$$

Now $(b)$ is obtained from Proposition 2.1. To prove $(a)$, since $\zeta_{k}^{m}$ is symmetric, it is sufficient to show that $\zeta_{k}^{m}\left(x_{1}-x_{2}\right)$ is divisible by $\left(x_{1}-x_{2}-p\right)$ for $-m \leq p \leq m$. If $p=0$, it is clear that

$$
\zeta_{k}^{m}\left(x_{1}-x_{2}\right)=-\ell \sum_{x_{2}}^{x_{1}} t^{k} g(t)^{\underline{m}} \Delta t
$$

is divisible by $x_{1}-x_{2}$. Now we assume $p>0$. To prove divisibility by $\left(x_{i}-x_{j}-p\right)$, we need to show that

$$
\sum_{x_{2}}^{x_{2}+p} t^{k} g(t)^{\underline{m}} \Delta t=0
$$

Actually, using (3.1), the left-hand side is equal to

$$
\sum_{t=x_{2}}^{x_{2}+p-1} t^{k} g(t) g(t-1) \cdots g(t-m+1) .
$$

For each $t=x_{2}+i(0 \leq i \leq p-1)$, clearly we have $g(t-i)=0$. Thus (4.1) holds. In the case $-m \leq p<0$, we need to consider $\sum_{x_{1}}^{x_{2}} \Delta t=\sum_{x_{1}}^{x_{1}-p} \Delta t$ instead of (4.1). The remaining arguments are similar. 


\subsection{A basis for the Shi arrangement}

To construct a basis for the Shi arrangement, we need the following. For $0 \leq k \leq \ell$ and $m \geq 0$, let

$$
g_{k}^{(m)}(t):=g(t) \frac{m-1}{1 \leq i<k} \prod_{1}\left(t-x_{i}+1\right) \prod_{k<i \leq \ell}\left(t-x_{i}-m+1\right) .
$$

We define the following vector field which is a discrete analogue of (2.3)

$$
\tau_{k}^{m}=\sum_{i, j=1}^{\ell}\left(\sum_{x_{i}}^{x_{j}} g_{k}^{(m)}(t) \Delta t\right) \partial_{i} .
$$

Using these vector fields, we can construct an explicit basis for the Shi arrangement.

Theorem 4.2. $\widetilde{\tau}_{1}^{m}-\widetilde{\tau}_{2}^{m}, \widetilde{\tau}_{2}^{m}-\widetilde{\tau}_{3}^{m}, \ldots, \widetilde{\tau}_{\ell-1}^{m}-\widetilde{\tau}_{\ell}^{m}, \theta_{0}$ and the Euler vector field $\theta_{E}:=z \partial_{z}+x_{1} \partial_{1}+$ $\cdots+x_{\ell} \partial_{\ell}$ form a basis for $D\left(c \operatorname{Shi}_{\ell}(m)\right)$.

Proof. The strategy is similar to the proof of Theorem 4.1. It is sufficient to prove the following:

(a) $\widetilde{\tau}_{1}^{m}-\widetilde{\tau}_{2}^{m}, \widetilde{\tau}_{2}^{m}-\widetilde{\tau}_{3}^{m}, \ldots, \widetilde{\tau}_{\ell-1}^{m}-\widetilde{\tau}_{\ell}^{m} \in D\left(c \operatorname{Shi}_{\ell}(m)\right)$.

(b) The restrictions $\left.\left(\widetilde{\tau}_{1}^{m}-\widetilde{\tau}_{2}^{m}\right)\right|_{z=0},\left.\left(\widetilde{\tau}_{2}^{m}-\widetilde{\tau}_{3}^{m}\right)\right|_{z=0}, \ldots,\left.\left(\widetilde{\tau}_{\ell-1}^{m}-\widetilde{\tau}_{\ell}^{m}\right)\right|_{z=0}$ form a basis of $D\left(\mathcal{B}_{\ell}, 2 m\right)$.

As in the proof of Theorem 4.1, (b) is obtained from Propositions 3.2 and 2.4.

To prove $(a)$, we need to show that for $1 \leq u<v \leq \ell$,

$$
\tau_{k}^{m}\left(x_{u}-x_{v}\right)=\ell \sum_{x_{u}}^{x_{v}} g_{k}^{(m)}(t) \Delta t
$$

is divisible by $\left(x_{u}-x_{v}-p\right)$ for any $1-m \leq p \leq m$. The case $p=0$ is obvious. We assume $0<p$. In this case, we need to show that

$$
\sum_{x_{v}}^{x_{v}+p} g_{k}^{(m)}(t) \Delta t=0 .
$$

By the formula (3.1), the left-hand side is equal to

$$
\sum_{s=0}^{p-1} g_{k}^{(m)}\left(x_{v}+s\right) .
$$

Suppose $0<p<m$. In this case, we have $m>1$. Then $g(t) \frac{(m-1)}{\underline{m}}$ vanishes when $t=x_{v}+s$, $0 \leq s \leq m-2$. The remaining case is $p=m$. Then $x_{u}=x_{v}+m$ and $t=x_{v}+m-1$. In this case, the product

$$
\prod_{1 \leq i<k}\left(t-x_{i}+1\right) \prod_{k<i \leq \ell}\left(t-x_{i}-m+1\right)
$$

is equal to zero. Indeed, if $v>k$, the factor $\left(t-x_{v}-m+1\right)$ vanishes. If $v \leq k$, then $u<k$. Then the factor $\left(t-x_{u}+1\right)$ vanishes.

The case $1-m \leq p<0$ is similar (simpler).

Remark 4.3. In [22], generalizations of integral formulas of Bandlow-Musiker are discussed. It would be interesting to consider discrete versions of such generalizations.

\section{Acknowledgements}

The authors thank Takuro Abe and Misha Feigin for many useful discussions on the topics. This work was partially supported by JSPS KAKENHI Grant Numbers JP18H01115, JP15KK0144. 


\section{References}

[1] Abe T., Enomoto N., Feigin M., Yoshinaga M., Free reflection multiarrangements and quasi-invariants, in preparation.

[2] Abe T., Suyama D., A basis construction of the extended Catalan and Shi arrangements of the type $A_{2}$, J. Algebra 493 (2018), 20-35, arXiv:1312.5524.

[3] Athanasiadis C.A., On free deformations of the braid arrangement, European J. Combin. 19 (1998), 7-18.

[4] Bandlow J., Musiker G., A new characterization for the $m$-quasiinvariants of $S_{n}$ and explicit basis for two row hook shapes, J. Combin. Theory Ser. A 115 (2008), 1333-1357, arXiv:0707.3174.

[5] Chalykh O.A., Veselov A.P., Commutative rings of partial differential operators and Lie algebras, Comm. Math. Phys. 126 (1990), 597-611.

[6] Edelman P.H., Reiner V., Free arrangements and rhombic tilings, Discrete Comput. Geom. 15 (1996), 307340 .

[7] Feigin M., Private communication.

[8] Feigin M., Veselov A.P., Quasi-invariants of Coxeter groups and $m$-harmonic polynomials, Int. Math. Res. Not. 2002 (2002), 521-545, arXiv:math-ph/0105014.

[9] Felder G., Veselov A.P., Action of Coxeter groups on $m$-harmonic polynomials and Knizhnik-Zamolodchikov equations, Mosc. Math. J. 3 (2003), 1269-1291, arXiv:math.QA/0108012.

[10] Gao R., Pei D., Terao H., The Shi arrangement of the type $D_{\ell}$, Proc. Japan Acad. Ser. A Math. Sci. 88 (2012), 41-45, arXiv:1109.1381.

[11] Orlik P., Terao H., Arrangements of hyperplanes, Grundlehren der Mathematischen Wissenschaften, Vol. 300, Springer-Verlag, Berlin, 1992.

[12] Postnikov A., Stanley R.P., Deformations of Coxeter hyperplane arrangements, J. Combin. Theory Ser. A 91 (2000), 544-597, arXiv:math.CO/9712213.

[13] Saito K., Theory of logarithmic differential forms and logarithmic vector fields, J. Fac. Sci. Univ. Tokyo Sect. IA Math. 27 (1980), 265-291.

[14] Saito K., Period mapping associated to a primitive form, Publ. Res. Inst. Math. Sci. 19 (1983), $1231-1264$.

[15] Saito K., On a linear structure of the quotient variety by a finite reflexion group, Publ. Res. Inst. Math. Sci. 29 (1993), 535-579.

[16] Saito K., Uniformization of the orbifold of a finite reflection group, in Frobenius Manifolds, Aspects Math., Vol. E36, Friedr. Vieweg, Wiesbaden, 2004, 265-320.

[17] Shi J.-Y., The Kazhdan-Lusztig cells in certain affine Weyl groups, Lecture Notes in Mathematics, Vol. 1179, Springer-Verlag, Berlin, 1986.

[18] Suyama D., A basis construction for the Shi arrangement of the type $B_{\ell}$ or $C_{\ell}$, Comm. Algebra 43 (2015), 1435-1448, arXiv:1205.6294.

[19] Suyama D., Terao H., The Shi arrangements and the Bernoulli polynomials, Bull. Lond. Math. Soc. 44 (2012), 563-570, arXiv:1103.3214.

[20] Terao H., Multiderivations of Coxeter arrangements, Invent. Math. 148 (2002), 659-674, arXiv:math.CO/0011247.

[21] Terao H., The Hodge filtration and the contact-order filtration of derivations of Coxeter arrangements, Manuscripta Math. 118 (2005), 1-9, arXiv:math.CO/0205058.

[22] Tsuchida T., On quasiinvariants of $S_{n}$ of hook shape, Osaka J. Math. 47 (2010), 461-485, arXiv:0807.1892.

[23] Yoshinaga M., The primitive derivation and freeness of multi-Coxeter arrangements, Proc. Japan Acad. Ser. A Math. Sci. 78 (2002), 116-119, arXiv:math.CO/0206216.

[24] Yoshinaga M., Characterization of a free arrangement and conjecture of Edelman and Reiner, Invent. Math. 157 (2004), 449-454.

[25] Yoshinaga M., Freeness of hyperplane arrangements and related topics, Ann. Fac. Sci. Toulouse Math. 23 (2014), 483-512, arXiv:1212.3523.

[26] Ziegler G.M., Multiarrangements of hyperplanes and their freeness, in Singularities (Iowa City, IA, 1986), Contemp. Math., Vol. 90, Amer. Math. Soc., Providence, RI, 1989, 345-359. 